J. Urol. Urogynäkol. AT 2021 · 28:29-33 https://doi.org/10.1007/s41972-021-00138-6 Angenommen: 7. Mai 2021

Online publiziert: 10. Juni 2021

○ Der/die Autor(en) 2021

\author{
Nathalie Garstka' · Shahrokh F. Shariat ${ }^{1,2,3,4,5,6,7}$ \\ ' Department of Urology, Comprehensive Cancer Center, Medizinische Universität Wien, Wien, Österreich \\ ${ }^{2}$ Institute for Urology and Reproductive Health, Sechenov Universität, Moskau, Russland \\ ${ }^{3}$ Department of Urology, Weill Cornell Medical College, New York, USA \\ ${ }^{4}$ Department of Urology, University of Texas Southwestern, Dallas, USA \\ ${ }^{5}$ Department of Urology, Second Faculty of Medicine, Charles Universität, Prag, Tschechien \\ ${ }^{6}$ Karl Landsteiner Institut für Urologie und Andrologie, Wien, Österreich \\ ${ }^{7}$ Division of Urology, Department of Special Surgery, Jordan University Hospital, The University of Jordan, \\ Amman, Jordanien
}

\title{
Das Harnblasenkarzinom der Frau
}

teressanterweise nur bei Leukämien, Gallengangkarzinomen und beim Blasenkarzinomen vor [3]. Laut einer SEERAnalyse mit 51.528 inkludierten Patienten liegt der Verlust an Lebenserwartung zum Zeitpunkt der Harnblasenkarzinomdiagnose bei Frauen mit 6,5 Jahren fast doppelt so hoch als bei Männern mit 3,9 Jahren und ist unabhängig des Stadiums höher [4].

\section{Risikofaktoren}

Die wichtigste Krankheitsursache für die Entstehung eines $\mathrm{BCa}$ ist das Rauchen. Sowohl für die Intensität und Dauer des Rauchens als auch für den lebenslangen Konsum von Tabak wurden Dosis-Wirkungs-Beziehungen nachgewiesen. Interessanterweise liegt die BCaInzidenz trotz gleich intensiven Zigarettenkonsums, welcher etwa $50 \%$ der Blasenkarzinome bedingt, mit 3,7:1 bei Männern höher als bei Frauen. Hier scheint hinsichtlich des Erkrankungsrisikos die individuelle genetische, aber auch geschlechtsspezifische Entgiftungskapazität über die Toxizität von kanzerogenen Substanzen zu entscheiden. Die meisten zigarettenassoziierten Kanzerogene entstehen im Organismus nach Verteilung und metabolischer Aktivierung. Die aktivierten Prokanzerogene werden verteilt und reagieren mit Proteinen und Nukleinsäuren [5]. Die Balance zwischen der metabolischen Aktivierung von prokanzerogenen Stoffen zu DNA- reaktiven Metaboliten und ihrer Detoxifikation hat einen wichtigen Einfluss auf das Krebsrisiko. Die Leber spielt eine entscheidende Rolle in der BCa-Entstehung, da Karzinogene primär in der Leber metabolisiert werden und renal über den Urin ausgeschieden werden. Die Karzinogene interagieren dann mit dem Urothel der Harnblase [6].

Einige Studien konnten zeigen, dass es geschlechtsspezifische Unterschiede hinsichtlich der Metabolitenverstoffwechselung gibt, die sich auf die jeweilige Blasenkrebsinzidenz auswirken können [7]. Ein Beispiel stellt der Abbau des in $\mathrm{Zi}$ garettenrauch vorkommenden aromatischen Amins 4-Aminobiphenyl dar. Ein Abbauweg von 4-Aminobiphenyl erfolgt in der Leber über die UDP-Glucuronosyltransferasen (UGT). Hierbei konnte gezeigt werden, dass es androgenabhängig zu einer geringeren Expression von UGT kommt [8].

Ein weiterer Faktor, welcher die BCaEntstehung geschlechtsspezifisch beeinflusst, sind die Sexualhormone. So konnte gezeigt werden, dass die urotheliale Tumorgenese durch Androgenrezeptoren aktiviert wird. Die Rolle von Östrogen in der BCa-Entstehung wurde erstmals im Jahre 1975 untersucht. Damals wurde kastrierten Ratten synthetisches Östrogen (Diethylstilbestrol) oder Testosteron verabreicht, woraufhin die Inzidenz von N-Butyl-N-(4Hydroxybutyl)nitrosamin-induziertem $\mathrm{BCa}$ in der Östrogengruppe sank und nern haben. Eine höhere Mortalität liegt bei Frauen in dieser Analyse in- 
in der Testosteronkohorte anstieg [9]. Ebenso konnte gezeigt werden, dass androgendeprivierte Ratten weniger synthetisch induzierte BCa entwickelten als Ratten ohne Hormonentzugstherapie [10]. Östrogene wirken sich im Gegensatz zu Testosteron hinsichtlich des Erkrankungsrisikos protektiv aus. Epidemiologische Untersuchungen ergaben, dass postmenopausale Frauen ein höheres BCa-Risiko haben als Frau vor der Menopause [11]. Ein höheres Alter (>15 Jahre) bei Einsetzen der Menstruation, Kombinationspräparate aus Östrogenen und Progesteron sowie Schwangerschaften sind ebenfalls mit einem geringeren Erkrankungsrisiko assoziiert [7]. Es scheint so zu sein, dass Östrogene sich protektiv auf eine Erkrankungsentstehung auswirken, hingegen aber im Falle eines vorhandenen $\mathrm{BCa}$ die Krankheitsprogression steigern.

\section{Histologie}

Die häufigste Tumorentität stellen mit $>95 \%$ Urothelkarzinome der Harnblase dar. Wesentlich seltener finden sich Plattenepithelkarzinome (ca. 2\%), Adenokarzinome (ca. 1\%) und vereinzelt Urachus-, kleinzellige und neuroendokrine Karzinome. Histologischen BCaVarianten sind mit einem höheren Rezidiv- und Progressionsrisiko assoziiert und somit biologisch aggressiver.

Ein wesentlicher Faktor, der die höhere Gesamtmortalität des BCa bei Frauen mitbedingt, ist das Vorliegen fortgeschrittener Tumorstadien bei der Erstdiagnose. Zwar entwickeln Frauen seltener als Männer ein $\mathrm{BCa}$, allerdings sind deren Tumoren, sofern es zu einer Erkrankung kommt, mit aggressiveren Eigenschaften verknüpft. In einer retrospektiven Analyse der „National Cancer Database“ wurden 27.525 BCa-Patienten untersucht, von denen $27,4 \%$ weiblich waren. Histologisch wurde bei Frauen mit $15,1 \%$ signifikant häufiger ein $\mathrm{BCa}$ nichturothelialen Ursprungs diagnostiziert als bei Männern mit 9,9\% $(p<0,001$; [12]).

Somit werden bei Frauen bei Erstdiagnose häufiger fortgeschrittene, „highgrade“ Urothelzellkarzinome und seltenere histologische Varianten eines $\mathrm{BCa}$ detektiert, die mit einem schlechteren Krankheitsoutcome verknüpft sind [1]. Es gibt mehrere Erklärungsansätze, weshalb Frauen bei der Erstdiagnose fortgeschrittene Tumoren aufweisen. Ein möglicher Grund liegt in der Anatomie und Beschaffenheit der weiblichen Harnblase. Der Detrusormuskel von Männern im typischen BCa-Erkrankungsalter um 65 Jahre ist häufig dicker als der von Frauen, was oftmals auf eine BPH-bedingte Miktionsveränderung und ein konsekutives Wachstum des Blasenmuskels zurückzuführen ist [13]. Durch die dünnere weibliche Harnblasenwand kommt es möglicherweise zu einem schnelleren extravesikalen Wachstum [1]. Durch die naturgemäß nicht vorhandene Denonvilliers-Faszie existiert eine ineffektivere Barriere, weshalb es zu einem leichteren lokalen Tumorwachstum per continuitatem kommen kann [1].

\section{Diagnosefindung}

Bei Frauen kommt es im Vergleich zu Männern häufiger zu einer Diagnoseverzögerung, was die fortgeschrittenen Tumorstadien bei Erstdiagnose miterklären kann. Die klinischen Symptome eines BCa sind bei beiden Geschlechtern gleich; hierzu zählen die schmerzlose Makrohämaturie und dysurische Beschwerden [14]. Laut verschiedener Untersuchungen ist das Intervall einer adäquaten Hämaturieabklärung bei Frauen deutlich länger als bei Männern [1]. Das liegt u. a. daran, dass es geschlechtsspezifische Unterschiede hinsichtlich der Konsultation eines Urologen gibt, sei es als direktes Aufsuchen durch die Patientinnen oder alsärztliche Überweisung z. B. durch Hausärzte. Etwa 47 \% der Männer gehen wegen einer Makrohämaturie direkt zu einem Urologen, aber nur $28 \%$ der Frauen $(p<0,001 ;[15])$. Unklare Miktionsbeschwerden wie Drangsymptomatik und Hämaturie werden oftmals einer hämorrhagischen Zystitis oder Detrusorüberaktivität zugeschrieben - an ein ursächliches Blasenkarzinom wird nicht oder verzögert gedacht. So wurden in einer retrospektiven Studie mit 168 neu diagnostizierten BCa-Patienten jeweils $47 \%$ Frauen 1 Jahr vor der Diagnose eines Urothelkarzinoms der Harnblase symp- tomatisch ohne weitere Abklärung behandelt, während es nur 19\% Männer waren $(p<0,05)$. Etwa 5-mal so viele Frauen erhielten $>3$ verschiedene Therapien zur Behandlung eines Harnwegsinfekts im Vergleich zu männlichen BCa-Patienten $(p<0,005 ;[14])$. Eine verzögerte Diagnostik, welche konsekutiv zu einer prognostisch ungünstigeren Diagnose fortgeschrittener BCa-Stadien führt, ist eine Erklärung für das schlechtere Outcome weiblicher BCa-Patientinnen.

\section{Therapieansprechen}

\section{NMIBC}

Hinsichtlich geschlechtsspezifischer Unterschiede bei der Behandlung des nichtmuskelinvasiven BCa (NMIBC) liegen in der Literatur diskrepante Ergebnisse vor. Eine Vielzahl von Studien konstatiert, insbesondere bei Patientinnen mit „high-grade“ $\mathrm{BCa}$, ein höheres Progressionsrisiko als bei Männern, unabhängig davon ob eine adjuvante intravesikale Immuntherapie mit BCG oder Chemotherapie wie z.B. Mitomycin C erfolgte [1]. In einer monozentrischen Studie, welche 146 Patienten inkludierte, konnte gezeigt werden, dass bei der Therapie eines „high-grade“ T1-BCa die Faktoren Carcinoma in situ (CIS) in der prostatischen Harnröhre und weibliches Geschlecht unabhängig mit einem signifikant höheren Risiko einhergehen, ein BCa-Rezidiv und eine Progression $\mathrm{zu}$ entwickeln oder an einem BCa zu versterben [16]. Eine Studie von Noon et al. konnte nachweisen, dass Patientinnen mit einem "high-risk“ NMIBC eine signifikant höhere krebsspezifische Mortalität aufwiesen als Männer $(p<0,01$; [17]). Interessanterweise konnte in einer multizentrischen Studie mit 916 inkludierten Patienten mit „highrisk" T1-BCa zwar der Faktor weibliches Geschlecht als ein unabhängiger Prädiktor für ein BCa-Rezidiv festgestellt werden; dies galt allerdings nicht für diejenigen Frauen, die eine BCG-Therapie nach ihrer TUR-B erhielten [18] Eine kürzlich veröffentlichte retrospektive Studie mit 2635 „high-grade“ pT1BCa-Patienten konnte zeigen, dass ein BCG-Ansprechen nur dann vom Ge- 
schlecht beeinflusst wird, sofern BCG nicht adäquat verabreicht wird [19]. Eine adäquate BCG-Therapie wurde hier definiert als eine mindestens 5-malig erfolgte intravesikale BCG-Induktion von 6 Applikationen, ergänzt durch eine Erhaltungstherapie bestehend aus mindestens 2 von 3 BCG-Dosen oder als eine Induktionstherapie (mindestens 5 von 6 Dosen) plus eine sekundäre Induktionstherapie, wobei hierbei mindestens 2 von 6 Dosen appliziert wurden. Insgesamt wurden 1056 Patienten nach dieser Definition adäquat mit BCG behandelt, davon 871/2170 Männer (40\%) und $185 / 465$ Frauen (40\%). Bei 52\% (454) der Männer und 54\% (99) der Frauen wurde ein Rezidiv festgestellt. Hierbei hatten $38 \%$ (328) der Männer und $40 \%$ (74) Frauen ein „high-grade“ Rezidiv. Eine BCa-Progression entwickelten nach der BCG-Therapie $17 \%$ (151) der Männer und $20 \%$ (37) der Frauen. In der Kohorte der adäquat BCG-therapierten konnte hinsichtlich der 3 Endpunkte (Rezidiv, „high-grade“ Rezidiv, Progression) kein signifikanter Einfluss des weiblichen Geschlechts nachgewiesen werden. In Zusammenschau scheint das schlechtere Outcome von Frauen nach BCGTherapie u.a. mit einem schlechteren Therapiemanagement zusammenzuhängen im Vergleich zu Männern, die eine adjuvante Therapie bei NMIBC erhalten.

\section{MIBC}

Wird ein muskelinvasives (MIBC) BCa diagnostiziert, besteht die Standardtherapie aus einer neoadjuvanten cisplatinhaltigen Chemotherapie, sofern der Patient dafür geeignet ist, und einer radikalen Zystektomie (RC) mit Lymphadenektomie. Zudem wird eine RC ebenso in selektiven Fällen von „high-risk“ NMIBC empfohlen. Der Großteil der publizierten Literatur hinsichtlich des Verlaufs eines muskelinvasiven BCa zeigt, dass Frauen auch nach Adjustierung prognoserelevanter Faktoren wie fortgeschrittene Tumorausbreitung und Lymphknotenstatus eine schlechteres Outcome haben als Männer [10, 18, 20]. In einer Auswertung des österreichischen Krebsregisters wurden zwischen 1983 und 2012 diagnostizierte BCa-Patienten aller Stadien 15 Jah-
J. Urol. Urogynäkol. AT 2021 · 28:29-33 https://doi.org/10.1007/s41972-021-00138-6

(c) Der/die Autor(en) 2021

\section{N. Garstka · S. F. Shariat}

\section{Das Harnblasenkarzinom der Frau}

\section{Zusammenfassung}

Frauen werden bis zur endgültigen Diagnosestellung eines Harnblasenkarzinoms (BCa) häufiger symptomatisch fehlbehandelt, wodurch es zu einer Diagnoseverzögerung kommt. Bei Frauen wird zum Zeitpunkt der Erstdiagnose eher ein fortgeschrittenes $\mathrm{BCa}$ festgestellt als bei Männern. Die krebsspezifische Mortalität ist bei Frauen insbesondere in den ersten 2 Jahren nach der Erstdiagnose höher als bei Männern. Die meisten Studien zeigen eine schlechtere Überlebensrate bei Frauen, welche aufgrund eines $\mathrm{BCa}$ eine radikale Zystektomie (RC) erhalten. Allerdings ist im Fall einer durchgeführten neoadjuvanten Chemotherapie (NAC) ein

besseres krebsspezifisches Überleben zu erwarten. Es gibt Hinweise, welche den Outcomeunterschied zwischen Männern und Frauen erklären; diese beziehen sich auf unterschiedliche Risikofaktoren, Diagnosestellungen, Behandlungen und hormonelle Faktoren. Weitere Studien sind notwendig, um diese Mechanismen genauer zu untersuchen. Dies gilt insbesondere auch für neuere systemische Therapie, wie Immuntherapeutika.

\section{Schlüsselwörter}

Blasenkarzinom · Geschlecht · Frauen . Diagnose $\cdot$ Prognose

\section{Bladder carcinoma in women}

\section{Abstract}

Until a definitive diagnosis of bladder carcinoma ( $\mathrm{BCa}$ ) is made, women are more likely to receive incorrect treatment for their symptoms, resulting in delayed diagnosis. They are also more likely to be diagnosed with advanced $\mathrm{BCa}$ at the time of initial diagnosis compared to men. Cancer-specific mortality is higher in women than in men, particularly in the first 2 years after initial diagnosis. Most studies show a poorer survival rate in women that receive radical cystectomy (RC) for BCa. However, in the case of neoadjuvant chemotherapy (NAC), better cancer-specific survival can be expected. There is evidence that explains the difference in outcome between men and women; this relates to different risk factors, diagnoses, treatments and hormonal factors. Further studies are needed to investigate these mechanisms in more detail. This applies in particular to newer systemic therapies such as immunotherapeutics.

Keywords

Bladder cancer - Gender - Women - Diagnosis . Prognosis re nachverfolgt [21]. Die inkludierten 27.773 Patienten zeigten ein Mann-zuFrau-Verhältnis von 3:1 auf. Zum Zeitpunkt einer RC wiesen Frauen ein weiter fortgeschrittenes Tumorstadium auf als Männer, und im Falle eines $\geq$ pT1$\mathrm{BCa}$ wiesen Frauen ein signifikant geringeres krebsspezifisches und Gesamtüberleben auf. In einer retrospektiven Analyse mit 458 BCa-Patienten, welche sich einer RC unterzogen, erhielten Frauen wesentlich häufiger eine inkontinente Harnableitung $(83,1 \%)$ im Vergleich zu Männern mit 60,2\%. Unabhängige Faktoren eines $\mathrm{M}+-$ Status waren weibliches Geschlecht und das Alter. Bluttransfusionen waren häufiger bei Frauen notwendig und Clavien-Dindo-Komplikationen
$>2$ traten ebenfalls häufiger bei Frauen als bei Männer auf (21,5\%: 14,4\%; [22]). In einer Analyse des norwegischen Krebsregisters wurden 15.129 BCa-Patienten mit unterschiedlichen Tumorstadien zwischen 1997 und 2011 untersucht. In dieser Analyse wurde ein geschlechtsabhängiges Sterberisiko festgestellt, welches, interessanterweise zeitlich limitiert war. Insbesondere bei MIBC-Patienten zeigte sich, dass innerhalb der ersten Erkrankungsjahre das Mortalitätsrisiko von Frauen größer war und sich dies nach 2 Jahren zu Ungunsten des männlichen Geschlechts umkehrte [23].

Dennoch gibt es hierzu inkonsistente Ergebnisse [1]. Heberling et al. [24] untersuchten in einer monozentrischen 
retrospektiven Studie insgesamt $1184 \mathrm{~Pa}$ tienten, die sich zwischen 1993 und 2015 wegen eines „high-risk“ NMIBC oder eines MIBC einer RC unterzogen hatten. Der Faktor weibliches Geschlecht war hier zwar mit einem erhöhten Risiko eines extravesikalen Stadiums vergesellschaftet, aber der Parameter weibliches Geschlecht war in der multivariaten Analyse kein unabhängiger Faktor für die krebsspezifische Mortalität (HR: 1,20, $95 \%$-KI: 0,94-1,54, $p=0,15)$. Die Verabreichung einer adjuvanten cisplatinhaltigen Chemotherapie war ebenfalls geschlechtsunabhängig. In einer weiteren kleineren monozentrischen Studie mit 259 inkludierten BCa-Patienten konnte nach RC kein signifikanter Unterschied zwischen männlichen und weiblichen $\mathrm{Pa}$ tienten festgestellt werden hinsichtlich Rezidivfreiheit, krebsspezifischem Überleben und Gesamtüberleben [25]. Dies konnte die Arbeitsgruppe um Patafio bestätigen. Hier gab es auch hinsichtlich der Applikation einer neoadjuvanten Chemotherapie (NAC) keinen geschlechtsspezifischen Unterschied [26]. In einer rezent veröffentlichten Metaanalyse mit 15 inkludierten Studien konnte gezeigt werden, dass Frauen, die eine NAC erhalten hatten, hinsichtlich der Entwicklung eines Rezidivs (gepoolte HR: 0,66; $95 \%$ KI: 0,44-0,98) und der krebsspezifischen Mortalität mehr profitierten als männliche BCa-Patienten mit NAC (gepoolte HR: 0,49, $95 \%$-KI: 0,29-0,81; [27]).

Zusammenfassend lässt sich feststellen, dass der Großteil der Studien zu diesem Thema eine schlechtere Prognose für Frauen nach Therapie eines MIBC nachweist, trotz adäquater Therapie. Eine Teilerklärung bietet die Tatsache, dass Frauen zum Zeitpunkt der potenziell kurativen Therapie ein weiter fortgeschrittenes Krankheitsstadium aufweisen.

\section{Metastasiertes BCa}

Wenige Studien beschäftigten sich mit dem geschlechtsspezifischen Outcome im Falle einer Therapie im metastasierten BCa-Erkrankungsstadium. Eine Analyse, welche die Ergebnisse von insgesamt 8 Phase-II- und -III-Studien zusammenfasste, welche eine cisplatinhaltige Erstlinienchemotherapie bei MIBC un- tersuchten, konnte eine gleichwertige Verträglichkeit bei Männern und Frauen feststellen und keine Unterschiede bzgl. des medianen Gesamtüberlebens (11,7 Monate bei Männern vs. 16,2 Monate bei Frauen; [28]). Konform dazu zeigte eine SEER-Analyse mit 3110 metastasierten BCa-Patienten keine geschlechtsspezifischen Unterschiede hinsichtlich des Überlebens [29]. Eine Studie der „National Cancer Database“ konnte feststellen, dass Frauen die gleichen lokalen Therapien im Falle eines metastasierten BCa-Stadium erhalten wie männliche Patienten [30]. Die Autoren verglichen hier metastasierte BCa-Patienten, die eine hochintensive Lokaltherapie bestehend aus RC oder Bestrahlungstherapie mit mindestens 50 Gy erhielten, und eine niedrigintensive Lokaltherapie (TURB oder nur Strahlentherapie < 50 Gy). Unabhängig des Geschlechts überlebten die Patienten länger, die eine hochintensive Therapie erhalten hatten [30].

\section{Korrespondenzadresse}

\section{Nathalie Garstka, BSC}

Department of Urology, Comprehensive Cancer Center, Medizinische Universität Wien

Währinger Gürtel 18-20, 1090 Wien, Österreich nathalie.garstka@meduniwien.ac.at

Funding. Open access funding provided by Medical University of Vienna.

\section{Einhaltung ethischer Richtlinien}

Interessenkonflikt. N. Garstka und S.F. Shariat geben an, dass kein Interessenkonflikt besteht.

Für diesen Beitrag wurden von den Autoren keine Studien an Menschen oder Tieren durchgeführt. Für die aufgeführten Studien gelten die jeweils dort angegebenen ethischen Richtlinien.

Open Access. Dieser Artikel wird unter der Creative Commons Namensnennung 4.0 International Lizenz veröffentlicht, welche die Nutzung, Vervielfältigung, Bearbeitung, Verbreitung und Wiedergabe in jeglichem Medium und Format erlaubt, sofern Sie den/die ursprünglichen Autor(en) und die Quelle ordnungsgemäß nennen, einen Link zur Creative Commons Lizenz beifügen und angeben, ob Änderungen vorgenommen wurden.

Die in diesem Artikel enthaltenen Bilder und sonstiges Drittmaterial unterliegen ebenfalls der genannten Creative Commons Lizenz, sofern sich aus der Abbildungslegende nichts anderes ergibt. Sofern das betreffende Material nicht unter der genannten Creative Commons Lizenz steht und die betreffende Handlung nicht nach gesetzlichen Vorschriften erlaubt ist, ist für die oben aufgeführten Weiterverwendungen des Materials die Einwilligung des jeweiligen Rechteinhabers einzuholen.

Weitere Details zur Lizenz entnehmen Sie bitte der Lizenzinformation auf http://creativecommons.org/ licenses/by/4.0/deed.de.

\section{Literatur}

1. Marks P, Soave A, Shariat SF, Fajkovic H, Fisch $M_{\text {, }}$ Rink M (2016) Female with bladder cancer: what and why is there a difference? Transl Androl Urol 5(5):668-682. https://doi.org/10.21037/tau.2016. 03.22

2. Sung $H$, Ferlay J, Siegel RL, Laversanne $M$ Soerjomataram I, Jemal A et al (2021) Global cancer statistics 2020: GLOBOCAN estimates of incidence and mortality worldwide for 36 cancers in 185 countries. CA Cancer J Clin. https://doi.org/ 10.3322/caac. 21660

3. Micheli A,CiampichiniR,OberaignerW, CiccolalloL, de Vries E, Izarzugaza l et al (2009) The advantage of women in cancer survival: an analysis of EUROCARE-4 data. Eur J Cancer 45(6):1017-1027. https://doi.org/10.1016/j.ejca.2008.11.008

4. Scosyrev E, Golijanin D, Wu G, Messing E (2012) The burden of bladder cancer in men and women: analysis of the years of life lost. BJU Int 109(1):57-62. https://doi.org/10.1111/j.1464410X.2011.10318.x

5. Peter Guengerich F, Avadhani NG (2018) Roles of cytochrome P450 in metabolism of ethanol and carcinogens. Adv Exp Med Biol 1032:15-35. https://doi.org/10.1007/978-3-319-98788-0 2

6. Zhang Y (2013) Understanding the gender disparity in bladder cancer risk: the impact of sex hormones and liver on bladder susceptibility to carcinogens. J Environ Sci Health C Environ Carcinog Ecotoxicol Rev 31(4):287-304. https:// doi.org/10.1080/10590501.2013.844755

7. Dobruch J, Daneshmand S, Fisch M, Lotan Y, Noon AP, Resnick MJ et al (2016) Gender and bladder cancer: a collaborative review of etiology, biology, and outcomes. Eur Urol 69(2):300-310. https://doi.org/10.1016/j.eururo.2015.08.037

8. Izumi K, Zheng Y, Hsu J-W, Chang C, Miyamoto H (2013) Androgen receptor signals regulate UDP-glucuronosyltransferases in the urinary bladder: a potential mechanism of androgeninduced bladder carcinogenesis. Mol Carcinog 52(2):94-102. https://doi.org/10.1002/mc.21833

9. Okajima E, Hiramatsu T, Iriya K, ljuin M, Matsushima $S$ (1975) Effects of sex hormones on development of urinary bladder tumours in rats induced by N-butyl-N-(4-hydroxybutyl) nitrosamine. Urol Res 3(2):73-79. https://doi.org/10.1007/ BF00256185

10. Fajkovic H, Halpern JA, Cha EK, Bahadori A, Chromecki TF, Karakiewicz Pl et al (2011) Impact of gender on bladder cancer incidence, staging, and prognosis. World JUrol 29(4):457-463. https://doi. org/10.1007/s00345-011-0709-9

11. McGrath M, Michaud DS, de Vivo I (2006) Hormonal and reproductive factors and the risk of bladder cancer in women. Am J Epidemiol 163(3):236-244. https://doi.org/10.1093/aje/kwj028

12. Krimphove MJ, Szymaniak J, Marchese M, Tully KH, D'Andrea D, Mossanen M et al (2019) Sex-specific differences in the quality of treatment of muscleinvasive bladder cancer do not explain the overall 
survival discrepancy. Eur Urol Focus. https://doi. org/10.1016/j.euf.2019.06.001

13. Rink M, Chun FK, Chromecki TF, Fajkovic H, Dahlem R, Fisch M et al (2012) Das fortgeschrittene Urothelkarzinom der Harnblase beim älteren Patienten. Ein Überblick über Inzidenz, Prognose und therapeutische Möglichkeiten. Urologe A 51(6):820-828. https://doi.org/10.1007/s00120011-2769-2

14. Henning $A$, Wehrberger $M$, Madersbacher $S$, Pycha A, Martini T, Comploj E et al (2013) Do differences in clinical symptoms and referral patterns contribute to the gender gap in bladder cancer? BJU Int 112(1):68-73

15. Johnson EK, Daignault S, Zhang Y, Lee CT (2008) Patterns of hematuria referral to urologists: does a gender disparity exist? Urology 72(3):498-502. https://doi.org/10.1016/j.urology.2008.01.086 (discussion 502-3)

16. Palou J, Sylvester RJ, Faba OR, Parada R, Peña JA, Algaba Fetal (2012) Female gender and carcinoma in situ in the prostatic urethra are prognostic factors for recurrence, progression, and diseasespecific mortality in T1G3 bladder cancer patients treated with bacillus Calmette-Guérin. Eur Uro 62(1):118-125. https://doi.org/10.1016/j.eururo. 2011.10.029

17. Noon AP, Albertsen PC, Thomas F, Rosario DJ, Catto JWF (2013) Competing mortality in patients diagnosed with bladder cancer: evidence of undertreatment in the elderly and female patients. Br J Cancer 108(7):1534-1540. https://doi.org/10. 1038/bjc.2013.106

18. Kluth LA, Fajkovic H, Xylinas E, Crivelli JJ, Passoni N, Rouprêt Met al (2013) Female gender is associated with higher risk of disease recurrence in patients with primary $\mathrm{T} 1$ high-grade urothelial carcinoma of the bladder. World J Urol 31(5):1029-1036. https://doi.org/10.1007/s00345-012-0996-9

19. D'Andrea D, Soria F, Grotenhuis AJ, Cha EK, Malats N, Di Stasi S et al (2021) Association of patients' sex with treatment outcomes after intravesical bacillus Calmette-Guérin immunotherapy for T1G3/HG bladder cancer. World J Urol. https://doi.org/10. 1007/s00345-021-03653-1

20. Mun D-H, Kimura S, Shariat SF, Abufaraj M (2019) The impact of gender on oncologic outcomes of bladder cancer. Curr Opin Urol 29(3):279-285. https://doi.org/10.1097/MOU. 0000000000000606

21. Waldhoer T, Berger I, Haidinger G, Zielonke N, Madersbacher S (2015) Sex differences of $\geq$ pT1 bladder cancer survival in Austria: a descriptive, long-term, nation-wide analysis based on 27,773 patients. Urol Int 94(4):383-389. https://doi.org/ $10.1159 / 000368418$

22. Gschliesser T, Eredics K, Berger I, Szelinger M, Klingler HC, Colombo T et al (2017) The impact of gender on tumour stage in in-house complications and choice of urinary diversion: results of the Austrian Cystectomy Registry. Urol Int 99(4):429-435. https://doi.org/10.1159/000477672

23. Andreassen BK, Grimsrud TK, Haug ES (2018) Bladder cancer survival: women better off in the long run. Eur J Cancer 95:52-58. https://doi.org/ 10.1016/j.ejca.2018.03.001

24. Heberling U, Koch R, Hübler M, Baretton GB, Hakenberg OW, Wirth MP et al (2018) Gender and mortality after radical cystectomy: competing risk analysis. Urol Int 101(3):293-299. https://doi.org/ 10.1159/000487445

25. Pichler R, Fritz J, Heidegger I, Oberaigner W, HorningerW, Hochleitner M (2017) Gender-related outcome in bladder cancer patients undergoing radical cystectomy. J Cancer 8(17):3567-3574 https://doi.org/10.7150/jca.21130

26. Patafio FM, Robert Siemens D, Wei $X$, Booth CM (2015) Is there a gender effect in bladder cancer? A population-based study of practice and outcomes. Can Urol Assoc J 9(7):269-274. https://doi.org/10. 5489/cuaj.2927

27. KimuraS, Iwata T, Abufaraj M, Janisch F, D’Andrea D, Moschini $M$ et al (2020) Impact of gender on chemotherapeutic response and oncologic outcomes in patients treated with radical cystectomy and perioperative chemotherapy for bladder cancer: a systematic review and meta-analysis. Clin Genitourin Cancer 18(2):78-87. https://doi.org/ 10.1016/j.clgc.2019.11.007

28. Haines L, Bamias A, Krege S, Lin C-C, Hahn N, Ecke TH et al (2013) The impact of gender on outcomes in patients with metastatic urothelial carcinoma. Clin Genitourin Cancer 11(3):346-352. https://doi.org/10.1016/j.clgc.2013.04.010

29. Pal SK, Lin YI, Yuh B, DeWalt K, Kazarian A, Vogelzang $\mathrm{N}$ et al (2015) Conditional survival in de novo metastatic urothelial carcinoma. PLoS ONE 10(8):e136622. https://doi.org/10.1371/journal. pone. 0136622

30. Seisen T, Sun M, Leow JJ,Preston MA, Cole AP, GelpiHammerschmidt F et al (2016) Efficacy of highintensity local treatment for metastatic urothelial carcinoma of the bladder: a propensity scoreweighted analysis from the national cancer data base. J Clin Oncol 34(29):3529-3536. https://doi. org/10.1200/JCO.2016.66.7352

Hinweis des Verlags. Der Verlag bleibt in Hinblick auf geografische Zuordnungen und Gebietsbezeichnungen in veröffentlichten Karten und Institutsadressen neutral.

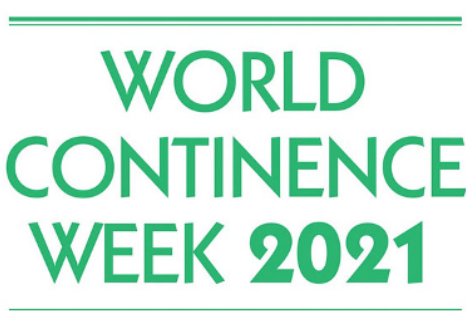

Welt-Kontinenz-Woche: Gut leben trotz Inkontinenz

Virtuelle Vorträge von Kontinenzund Stomaberaterinnen

Die Welt-Kontinenz-Woche ist eine globale Initiative der World Federation for Incontinence and Pelvic Problems (WFIPP) gemeinsam mit der International Continence Society (ICS). Ziel der jährlichen Aktionswoche ist, betroffene Menschen über die Möglichkeiten der Vorbeugung sowie Behandlung zu informieren und das schambesetzte Leiden ein Stück weit aus dem Tabu zu holen.

Die Medizinische Kontinenzgesellschaft Österreich (MKÖ) war im Juni aktiv und demonstrierte im Rahmen virtueller Vorträge von Kontinenz- und Stomaberaterinnen unter anderem, was Patienten selbst zur Verbesserung der Kontinenzsituation beitragen können. Im Fokus standen die zahlreichen Fragen des Alltags mit Inkontinenz und das Tabu, das damit verbunden ist: Kann man trotz einer Inkontinenz am sozialen Leben teilnehmen oder sogar reisen? Welche Hilfsmittel stehen zur Verfügung, sodass man wieder einen weitgehend normalen Alltag leben kann? Was kann man selbst dazu tun, um die Kontinenz zu erhalten bzw. zu verbessern?

MKÖ-Präsidentin OÄ Dr. Michaela Lechner: „Unser Ziel ist es, mehr Bewusstsein für das immer noch stark tabuisierte Thema Inkontinenz zu schaffen und Informationen anzubieten, die zur Verbesserung der Lebensqualität von Patienten beitragen."

Alle Videos gibt's im YouTube-Kanal der MKÖ: www.youtube.com/kontinenz

Medizinische Kontinenzgesellschaft Österreich 